by a 'Sylvania Blacklite Blue' fluorescent lamp for examining the chromatograms. By this method, one can readily detect $0.05 \mu \mathrm{gm}$. of a catecholamine.

For the quantitative evaluation of the fluorescence considerably more elaborate methods have been evolved. Instead of spraying the chromatograms, the dipping technique has been used. The strip is dipped first into a 1 per cent solution of iodine in carbon tetrachloride, blotted, and before it is completely dried, dipped into a 1 per cent solution of ethylenediamine in isopropyl alcohol, and again blotted. The strip is then hung to dry at room temperature. The fluorescence of epinephrine appears within 30 min. whereas that due to nor-epinephrine and other catecholamines is much slower. The maximal difference between background and epinephrine fluorescence occurs in about $24 \mathrm{hr}$. At that time, the strips were scanned by means of a mechanical device which pulls the strip past the beam of a Beckman $D U$ photometer equipped with a photomultiplier tube. The output of the photocell is recorded on a strip chart recorder. The 417-m $\mu$ light produces the maximal fluorescence. The exciting light is removed from the beam by a Corning colour filter 4015 . The areas under the peaks are related to amounts of catecholamines by the use of a standard curve ${ }^{2}$.

For example, 1 ugm. quantities of epinephrine were placed on four one-inch strips of Whatman 1 paper and chromatographed using $t$-butanol/formic acid/water $(40: 1: 10)$ as the solvent. After $40 \mathrm{hr}$. (strips were cut so that the solvent dripped from the end) the strips were dried and treated as described above. Under these conditions, areas of 132, 130, 126 and $138 \mathrm{~cm}^{2}$ were measured on the strip chart record of the fluorescence on these chromatograms. (It should be noted that the absolute areas have no significance as they are determined by the sensitivity of the photocell, the slit width, the brightness of the exciting beam, etc., as well as the amount of material on the chromatogram. Thus, it is necessary to compare the areas from the scans on unknown chromatograms with those on known amounts, using the identical conditions of scanning.) Using the most sensitive techniques (brightest exciting light, most sensitive position of the photocell) one can estimate $0.03 \mathrm{\mu gm}$.; for example, four chromatograms, prepared in the above way with $0.3 \mu \mathrm{gm}$., gave areas of 33,41 , 31 and $27 \mathrm{~cm}^{2}$.

Biochemical Research Laboratory,

The Dow Chemical Company, Midland, Michigan.

'Malherbe, H., and Bone, A. D., Biochem. J., 51, 311 (1952).

2 Brown, J. A., and Marsh, M. M., Anal. Chem., 25, 1865 (1953).

\section{Coding Problem in Proteins}

IN a previous publication ${ }^{1}$ a hypothesis was proposed to the effect that if a nucleic acid serves as a template for the linear array of amino-acids in a protein, it prescribes only the positions to be occupied by the aromatic amino-acids in that protein. It was postulated that the planar rings of the aromatic amino-acids would be differentially bound in cavities formed by two adjacent bases in such a way that the aromatic amino-acids with only six-membered rings (group 1) would occupy the space between two pyrimidines and those with five-membered rings (group 2) would occupy that between two purines. This hypothesis imposes certain restrictions on the arrangement of these amino-acids in proteins. It requires that, sandwiched between a pair of aromatic amino-acids belonging to the same group, there will be either none or an even number of non-aromatic amino-acids, and that an odd number will separate aromatic amino-acids of different groups.

Much progress has been made in recent years in the elucidation of amino acid sequences in proteins, affording further test for the restrictions imposed by the hypothesis. Phenylalanine does not conform to this scheme, and its position must therefore be specified in some other manner. However, with regard to the other aromatic amino-acids, tyrosine (group 1) and histidine and tryptophan (group 2), the spacing between the aromatic pairs is of the order required by the hypothesis in esch of the twelve cases reported where such a test is possible. These sequences are listed in Table 1. If any arrangement of aromatic amino-acids were permissible, the probability of obtaining this result attributable to chance alone would be very small.

Table 1. amino-acid Sheunnogs involving a Pair of the Aromatio AMINO-ACIDS

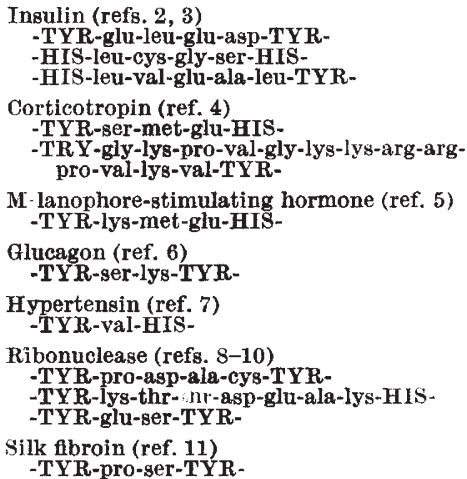

The non-randomness in the arrangement of the aromatic amino-acids offers support for the proposed coding system, although the lack of conformation of phenylalanine is a serious obstacle that must be overcome. It should be mentioned that phenylalanine stood apart from the other aromatic amino-acids even in the three protein sequences examined in the earlier paper. The observed restrictions suggest that the conclusion of Brenner"12, that "all amino sequences are likely to be found and that it will not be possible to effect a 'decoding' by discovering restrictions in sequences", may not be valid.

Biology Division,

Drew Schwartz

Oak Ridge National Laboratory*, Oak Ridge, Tennessee.

* Oporated by Union Carbide Nuclear Co. for the U.S. Atomic Energy Commission.

${ }^{1}$ Schwartz, D., Proc. U.S. Nat. Acad. Sci., 41, 300 (1955).

${ }^{2}$ Sanger, F., and Thompson, E. O. P., Biochem. J., 52, iil (1952).

${ }^{3}$ Sanger, F., and Tuppy, H., Biochem. J., 49, 481 (1951).

${ }^{4}$ Bell, P. H., J. Amer. Chem. Soc., 76, 5565 (1954).

${ }^{5}$ Harris, J. I., and Roos, P., Nature, 178, 90 (1956).

Bromer, W. W., Senn, L. G., Straub, A., and Behrens, O. K. J. Amer. Chem. Soc., 78, 3858 (1956).

7 Elliot, D. F., and Peart, W. S., Nature, 177, 527 (1956).

${ }^{3}$ Hirs, C. H. W., Stein, W. H., and Moore, S., J. Biol. Chem., 221, 151 (1956).

${ }^{9}$ Anfinsen, C. B., and Redfleld, R. R., "Adv. in Protein Chem.", 11, $1(1956)$

${ }^{17}$ Hirs, C. H. W., Fed. Proc., 16, 196 (1957).

${ }_{11}$ Abderhalden, E., and Bahn, A., Z. physiol. Chem., 219, 72 (1933).

${ }^{32}$ Brenner, S., Proc. U.S. Nat. Acad. Sci., 43, 687 (1957). 